\title{
The translation of substance dependence criteria to food-related behaviors: different views and interpretations
}

\author{
Adrian Meule* and Andrea Kübler \\ Department of Psychology I, University of Würzburg, Würzburg, Germany \\ ${ }^{*}$ Correspondence: adrian.meule@uni-wuerzburg.de \\ Edited by: \\ Marco Diana, University of Sassari, Italy \\ Reviewed by: \\ Hisham Ziauddeen, University of Cambridge, UK
}

\section{A commentary on}

Obesity and the brain: how convincing is the addiction model?

by Ziauddeen, H., Farooqi, I. S., and Fletcher, P. C. (2012). Nat. Rev. Neurosci. 13, 279-286.

In recent years, a food addiction model of obesity and overeating is gaining more and more popularity. A remarkable increase of the number of publications containing the topic food addiction has been observed since 2009 (Gearhardt et al., 2011). The concept of food addiction proposes that there are similarities - both neurobiological and behavioral-between obesity (or overeating) and substance dependence and suggests that hyperpalatable foods could have an addiction potential (Gearhardt et al., 2011). One of the most highlighted arguments is that DSM-IV substance dependence criteria also apply to overeating provided they are modified with references to binge eating (Cassin and von Ranson, 2007). A reward deficiency syndrome is also considered reflected by a downregulation of striatal D2-receptor availability and concurrent hypersensitivity to palatable food-cues in obese individuals (Kenny, 2011).

Ziauddeen et al. (2012) recently presented a critical evaluation of this food addiction model of obesity and overeating. Some key conclusions drawn in this article are that a vast majority of obese individuals would not show a convincing behavioral or neurobiological profile that resembles addiction and that the evidence for an overlap with addiction would be inconsistent and weak even when the food addiction model would be refined to obese individuals with binge eating disorder (BED).

Specifically, the authors conclude that "food addiction may prevail in non-obese and not-yet-obese individuals" and that "obesity, particularly when assessed solely cross-sectionally by body-mass index, will be an unsatisfactory phenotype for food addiction" (Ziauddeen et al., 2012, p. 281). In our opinion, Ziauddeen et al. (2012) correctly infer that not all obese individuals are food addicted and that food addiction does rather relate to binge eating behaviors, but they miss the fact that most researchers in this area would agree with this idea. For example, many researchers do mention that food addiction is not restricted to obesity, but also occurs in normal-weight samples, e.g., in patients with bulimia nervosa in particular (e.g., Davis and Carter, 2009; Gearhardt et al., 2009a; Broft et al., 2011; Meule, 2011; Speranza et al., 2012; Umberg et al., 2012). Furthermore, recent studies using the Yale Food Addiction Scale (YFAS) show that only a subset of obese individuals receive a "diagnosis" of food addiction (between 25 and 42\%; Davis et al., 2011; Meule et al., in press a,b) supporting the notion of the authors of a better behavioral profiling of investigations ( $p$. 284). Nonetheless, obese individuals are well-suited for research on food addiction because symptomatology is much more prevalent as compared to non-obese samples (Meule, 2011).

Another conclusion of the authors is that "the DSM-IV criteria for substance dependence translate poorly to food-related behaviors" (p. 281). The proposed food addiction equivalents of substance dependence criteria presented by the authors are based on articles by Volkow and O'Brien (2007) and Gearhardt et al. (2009a). We argue that those criteria require further examination.

Firstly, Ziauddeen et al. (2012) acknowledge that some criteria are easily applied to eating behavior, those are (1) a persistent desire or unsuccessful attempts to cut down (2) larger amounts consumed than intended and (3) continued use despite physical or psychological problems. They do also suggest, that these criteria require "the application of severity and impairment thresholds to be meaningful" (p. 280), although such thresholds do not exist for substance dependence. Dieting attempts, occasional overeating, and unhealthy eating despite problems apply to many people regardless of food addiction symptomatology that is why, as in substance dependence, not a single criterion suffices to receive the "diagnosis" food addiction. Specific severity thresholds may be difficult to establish because of a lack of a clear addictive agent associated with food addiction. However, the only currently available measure for establishing a diagnosis of food addiction, the YFAS, includes items for the assessment of distress or a clinically significant impairment because of eating and food addiction is only diagnosed when this criterion is met (Gearhardt et al., 2009b; Meule et al., in press b).

Secondly, the authors argue that the criteria of tolerance and neglecting or abandoning important activities in favor of substance-related activities have no convincing equivalents in relation to eating behavior. They define tolerance such that increasing amounts of food are required to reach satiety and that important activities are given up due to fear of rejection because of obesity. These definitions, however, differ from the definitions used in current research. For instance, the YFAS defines tolerance such that increasing amounts of food are necessary to reduce negative emotions or increase pleasure or that the same amount of food does no longer enhance mood (Gearhardt et al., 2009b; Meule et al., in press b). With regard to neglecting important activities, the authors propose that "a strict equivalent would require 
engagement in eating to the exclusion of other activities" (p. 280) and this suggestion exactly matches how this criterion is assessed with the YFAS (e.g., "There have been times when I consumed certain foods so often or in such large quantities that I started to eat food instead of working, spending time with my family or friends, or engaging in other important activities or recreational activities I enjoy"; Gearhardt et al., 2009b; Meule et al., in press b).

Thirdly, the authors argue that there is no convincing evidence for withdrawal symptoms when restricting certain foods and that the criterion for great time effort in relation to eating is difficult to apply because of the high availability of foods. It is true that those criteria do not apply to food as much as do the other criteria. However, in recent studies that used the YFAS, a subset of individuals with obesity or BED meet these criteria (Meule, 2011; Gearhardt et al., 2012; Meule et al., in press b). For example, approximately $55 \%$ of obese participants with BED felt that they often experience withdrawal symptoms which were defined as an elevated desire to consume certain foods or agitation, anxiety, or other physical symptoms when cutting down or stop eating certain foods (Gearhardt et al., 2012). Studies which objectively measure and establish the presence of such withdrawal symptoms and, most importantly, distinguish between withdrawal symptoms in relation to specific foods as compared to similar reactions due to plain caloric deficit are desperately needed.

To conclude, Ziauddeen et al. (2012) correctly point out that food addiction does not apply to all, but is also not restricted to, obese individuals and that there is a need for more precise definitions and future investigations to strengthen - or reject - the food addiction model. We agree unequivocally with these statements. However, the definitions of food addiction equivalents of substance dependence criteria based on Volkow and O'Brien (2007) and Gearhardt et al. (2009a) and their interpretations are debatable. An evaluation of the applicability of substance dependence criteria to overeating or obesity crucially depends on how food addiction symptoms are defined. There is a lack of agreement among researchers particularly with regard to what constitute tolerance or withdrawal symptoms and reasons underlying the reduction of important activities. In the present article, we have outlined that recent questionnaire studies, in which definitions of some food addiction symptoms differed from those presented by Ziauddeen et al. (2012), do indeed find that a substantial subset of obese individuals meet those addiction criteria (Merlo et al., 2009; Davis et al., 2011; Gearhardt et al., 2012; Lent and Swencionis, 2012; Meule et al., in press a). As a result, the food addiction model cannot be rejected when those definitions of food addiction symptoms are used. Future investigations relying on multimodal measures including physiological parameters, e.g. blood glucose level during withdrawal or longitudinal studies assessing the development of food intake over several time points, are required to further validate those subjectively gained insights.

\section{ACKNOWLEDGMENTS}

A. Meule is supported by a grant of the research training group 1253/2 which is supported by the German Research Foundation (DFG) by federal and Länder funds.

\section{REFERENCES}

Broft, A. I., Berner, L. A., Martinez, D., and Walsh, B. T. (2011). Bulimia nervosa and evidence for striatal dopamine dysregulation: a conceptual review. Physiol. Behav. 104, 122-127.

Cassin, S. E., and von Ranson, K. M. (2007). Is binge eating experienced as an addiction? Appetite 49, 687-690.

Davis, C., and Carter, J. C. (2009). Compulsive overeating as an addiction disorder. A review of theory and evidence. Appetite 53, 1-8.

Davis, C., Curtis, C., Levitan, R. D., Carter, J. C., Kaplan, A. S., and Kennedy, J. L. (2011). Evidence that 'food addiction' is a valid phenotype of obesity. Appetite 57, 711-717.

Gearhardt, A. N., Corbin, W. R., and Brownell, K. D. (2009a). Food addiction - an examination of the diagnostic criteria for dependence. J. Addict. Med. 3, 1-7.
Gearhardt, A. N., Corbin, W. R., and Brownell, K. D. (2009b). Preliminary validation of the Yale Food Addiction Scale. Appetite 52, 430-436.

Gearhardt,A. N., Davis, C., Kuschner, R., and Brownell, K. D. (2011). The addiction potential of hyperpalatable foods. Curr. Drug Abuse Rev. 4, 140-145.

Gearhardt, A. N., White, M. A., Masheb, R. M., Morgan, P. T., Crosby, R. D., and Grilo, C. M. (2012). An examination of the food addiction construct in obese patients with binge eating disorder. Int. J. Eat. Disord. 45, 657-663.

Kenny, P. J. (2011). Reward mechanisms in obesity: new insights and future directions. Neuron 69, 664-679.

Lent, M. R., and Swencionis, C. (2012). Addictive personality and maladaptive eating behaviors in adults seeking bariatric surgery. Eat. Behav. 13, 67-70.

Merlo, L. J., Klingman, C., Malasanos, T. H., and Silverstein, J. H. (2009). Exploration of food addiction in pediatric patients: a preliminary investigation. J. Addict. Med. 3, 26-32.

Meule, A. (2011). How prevalent is "food addiction"? Front. Psychiatry 2:61. doi: 10.3389/fpsyt.2011.00061

Meule, A., Heckel, D., and Kübler, A. (in press a). Factor structure and item analysis of the Yale Food Addiction Scale in obese candidates for bariatric surgery. Eur. Eat. Disord. Rev.

Meule,A., Vögele, C., and Kübler,A. (in press b). [German translation and validation of the Yale Food Addiction Scale]. Diagnostica.

Speranza, M., Revah-Levy, A., Giquel, L., Loas, G., Venisse, J.-L., Jeammet, P., and Corcos, M. (2012). An investigation of Goodman's addictive disorder criteria in eating disorders. Eur. Eat. Disord. Rev. 20, 182-189.

Umberg, E. N., Shader, R. I., Hsu, L. K., and Greenblatt, D. J. (2012). From disordered eating to addiction: the "food drug" in bulimia nervosa. J. Clin. Psychopharmacol. 32, 376-389.

Volkow, N. D., and O'Brien, C. P. (2007). Issues of DSM-V: should obesity be included as a brain disorder? Am. J. Psychiatry 164, 708-710.

Ziauddeen, H., Farooqi, I. S., and Fletcher, P. C. (2012). Obesity and the brain: how convincing is the addiction model? Nat. Rev. Neurosci. 13, 279-286.

Received: 02 May 2012; accepted: 11 June 2012; published online: 26 June 2012.

Citation: Meule A and Kübler A (2012) The translation of substance dependence criteria to food-related behaviors: different views and interpretations. Front. Psychiatry 3:64. doi: $10.3389 /$ fpsyt.2012.00064

This article was submitted to Frontiers in Addictive Disorders, a specialty of Frontiers in Psychiatry.

Copyright (c) 2012 Meule and Kübler. This is an openaccess article distributed under the terms of the Creative Commons Attribution Non Commercial License, which permits non-commercial use, distribution, and reproduction in other forums, provided the original authors and source are credited. 